\title{
Vega spacecraft reveal more dust than expected
}

\section{Moscow}

THE successful flight of the two Soviet space probes past the nucleus of Halley's comet showed that even from a distance of less than $8,000 \mathrm{~km}$ the nucleus of the comet was mostly obscured by dust. Estimates of the quantity of water being evaporated from the comet last week, a month after perihelion, range up to $2 \times$ $10^{30}$ molecules per second. The three separate dust recorders in the first Vega package have shown that the mass of dust being lost is roughly a sixth that of the water. In round numbers, the water loss on 6 March was 30 tonnes an hour, that of dust roughly 5 tonnes an hour.

One practical consequence is that images from the two cameras on Vega 1 were unable to distinguish the nucleus of the comet within its dust cocoon. By the time of the encounter by Vega 2 on 10 March, the quantity of dust had fallen by a factor of two, for reasons not necessarily connected with the increasing distance of the comet from the Sun. But the camera images on this occasion are not as easily interpreted because of a pointing error, corrected only 10 minutes before the encounter. Even so, the Hungarian team working on the processing of images says it has extracted the information that the nucleus has a dumbbell shape and is less than $10 \mathrm{~km}$ across at the maximum.

The dust measurements have provided most of the surprises. Both Vega craft encountered the main dust sheet at about $100,000 \mathrm{~km}$ from the nucleus of the comet, in line with the expectation that the dust is responsible for the coma caused by the scattering of solar radiation. But some of the experimenters have been surprised to find recordings of dust particles from the comet as far away as $150,000 \mathrm{~km}$.

Others say it is surprising that the dust recorders show the appearance of fine dust $\left(10^{-15}\right.$ grams or smaller $)$ before that of more substantial grains. On the present view of the formation of the coma and the cometary tail, dust is carried from the solar side of the nucleus by the stream of evaporated water molecules until driven away from the Sun by radiation pressure, which leads to the prediction that larger grains should form the outermost sheath of the coma and the tail.

The sheer size of the dust particles has also been surprising. The Soviet originators of the photon experiment, an ingenious device by which the holes caused by dust particles penetrating the thin outer shell of the Vega spacecraft are measured optically and the accompanying plasma jet is detected electronically in such a way as to provide both the energy and momen- tum of the incident particle, say the largest particle recorded by Vega 2 had a mass of $2 \times 10^{-5}$ grams. It left a hole in the outer skin nearly half a centimetre across.

The approach to the comet by both spacecraft has also provided a wealth of information about the transition between the region dominated by the solar wind and that in which ions from the comet are predominant. At this stage, it is not clear

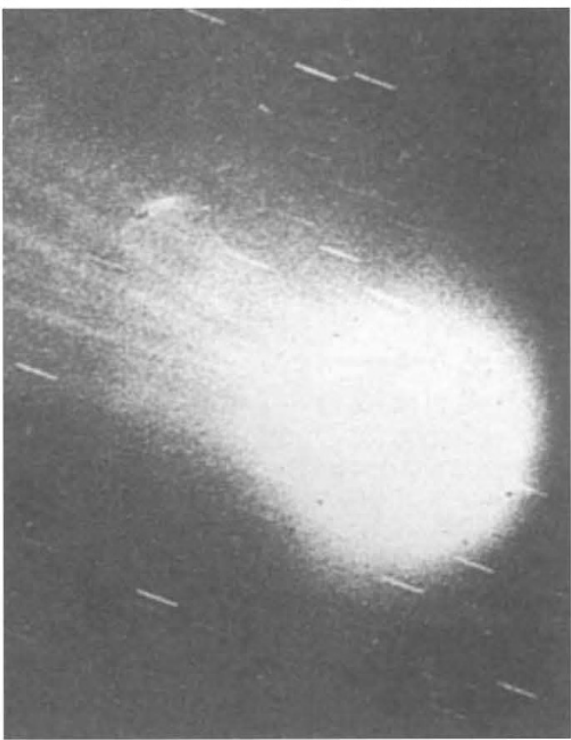

Image of comet Halley on 17 December 1985 , taken with a $1-\mathrm{m}$ reflector at the Alma-Ata Observatory by K.I. Churyumurov and Rspayev. North is up and East to the left.

whether either of the spacecraft detected the expected bow shock, representing the interaction between the solar wind and the cometary environment. Vega 1 may have passed through the bow shock before the instruments were switched on just over three hours before the encounter on 6 March; alternatively, the transition region may be more diffuse than expected.

At the second encounter on 10 March, both the intensity of the solar wind and the concentration of gas and dust from the comet were reduced by a half. The plasma detectors on Vega 2 have clearly shown how, on the inward passage towards the comet, ions (mainly protons) characteristic of the solar wind are gradually replaced by heavier ions, chiefly those of water and its photolysis products, between $150,000 \mathrm{~km}$ and $50,000 \mathrm{~km}$ from the nucleus.

Magnetic field measurements in the hours before the two encounters, essentially with similar results, also show the steady transition from the interplanetary to the cometary environment. On both occasions, the magnetic field increased (but with an apparent periodicity which is not yet explained) from the
$12-15 \mathrm{nT}\left(1 \mathrm{nT}=10^{-4}\right.$ gauss $)$ characteristic of the interplanetary solar wind regime to $75 \mathrm{nT}$ within the cometary environment.

Some of the most striking data are those from the time-of-flight mass spectrometer designed at the Max-Planck-Institut für Kernphysik at Heidelberg, built with French funds and operated jointly with Soviet specialists. The instrument provides an instant mass spectrogram of the content of the plasma produced by the impact of individual dust particles on a silver target. Vega 1 provided more than 1,000 records, Vega 2 nearly half as many. Although many particles appear to have been composed largely of light elements with atomic number less than 20 , there is a substantial fraction with components of atomic number between 30 and 50 . The interest will be to see whether the second group shows the presence of silicon and iron, expected constituents of primordial dust.

The two encounters have also provided an extremely accurate determination of the orbit of Halley's comet, chiefly through the work of the Pathfinder project designed so as to provide the basis for guiding the European spacecraft Giotto to its encounter with Halley's comet on Thursday this week. On the basis of the data from Vega 1, the Pathfinder project says that it has been able to define the position of the comet in its orbit with an uncertainty of only $15 \mathrm{~km}$.

One of the team responsible says that ground-based observations in the past few years have refined the orbital elements of the comet by a factor of 100 , while triangulation based on accurate radiointerferometric measurements of the position of the Vega spacecraft and a knowledge of their pointing angles towards the Halley nucleus have made possible a further refinement by a factor of 30 .

Elaborate arrangements have been made to communicate this information to those at the Darmstadt centre of the European Space Agency in West Germany, from where the Giotto encounter is being controlled. It is especially relevant that the uncertainty of the position of the comet along its orbit has been reduced from several hundred kilometres to a few tens of kilometres, given the ambition to send Giotto within $500 \mathrm{~km}$ of the nucleus.

A final decision about the direction of Giotto was due to be made on Tuesday this week. According to Dr RogerMaurice Bonnet, the French scientific director of the Giotto project, the decision is certain to have been difficult. Five of the nine experiments on Giotto would prefer to get even closer to the nucleus than 500 $\mathrm{km}$, but the other four wish to survive beyond the closest approach while two would like to survive by several days. One complicating factor is that nobody at 
present understands why there has been such a rapid decrease of dust production in the three days between the two Vega encounters.

Dust appears to have played havoc with several of the Vega experiments. A lowfrequency plasma detector, based on an antenna stretched between one of the booms projecting from the spacecraft and the solar panels, went out of action just before the first encounter, to the resigned annoyance of the group responsible. On the less successful encounter of Vega 2, several instruments went out of action before the encounter. One speculation is that the spacecraft may have become electrically charged in such a way as to affect the operation of instruments requiring a stable reference voltage.

The encounter of Vega 2 was also complicated by a pointing error, which for most of the approach had the cameras locked on to a bright patch of scattered sunlight offset from the nucleus of the comet. The most serious consequence was that the automatic microprocessor-based program relating the exposure of each frame to the brightness of the aiming point consistently over-exposed the nucleus. In a nail-biting emergency, the error was corrected only ten minutes before the encounter with the help of a back-up guidance system.

The general opinion of experimenters and managers is that the project has been outstandingly successful. Those gathered at the Institute of Space Science of the Soviet Academy of Science, which has mounted the exercise, have been quick to pay tribute to the international collaboration on which the project has been based.

Collaborators come from France and West Germany in Western Europe and from Czechoslovakia, Hungary and Yugoslavia in Eastern Europe. Although the United States is not formally involved in a project mounted when relations between the Soviet Union and the United States were in the doldrums, Professor J.A. Simpson from the University of Chicago has flown a novel dust detector on the identical Vega spacecraft at the invitation of Professor R.Z. Sagdeev, the director of the Soviet project and the institute at which it is based.

By common consent, the Deep Space Tracking Network of the US National Aeronautics and Space Administration (NASA) had also provided invaluable assistance both during the preliminary encounters of the Vega spacecraft with the gravitational field of Venus at the end of last year and in refining the trajectory of the vehicles.

Sagdeev recalls the Jet Propulsion Laboratory in California calling up one night with the question why one of the two Vegas had apparently vanished from the radio sky.

Halley's comet

\section{Telling the truth in real time}

\section{Moscow}

As this monumental city last Wednesday watched its four-month covering of snow turn to grey slush, the builders of the first of four probes to Halley's comet were celebrating (mostly in the abstemious Gorbachev style) their successful first look at that eroding dirty snowball in the sky. But the more striking achievements may be the informality of the international collaboration on which the Vega project was based and the frankness with which all the details of the project were displayed.

To say that the outcome confirms the dirty snowball picture of a comet, that of a lump of frozen water with embedded dust, true though it might be, would be to diminish the occasion. Getting within $8,000 \mathrm{~km}$ of an object nine light-minutes away may be child's play these days, while Voyager has shown that instruments can be made to function at least a decade after being built, but who can suppress childish pleasure that five years of planning for an hour or so of proximity to Halley should, in the end, succeed?

There were, in fact, two Vegas and thus two close encounters, on Wednesday last week and on Sunday. As launched (in December 1984), the two spacecraft were identical. Each of them dropped a balloon into the atmosphere of Venus before being deflected towards the retrograde orbit of Halley, still last week between the orbits of the Earth and Venus (but with the Earth on the other side of the Sun). At the Soviet Academy of Science's Institute of Space Sciences, the interval was spanned by a kind of perpetual polyglot symposium interrupted only by visits to other laboratories and the need for lunch.

Strictly, there have been two on-going

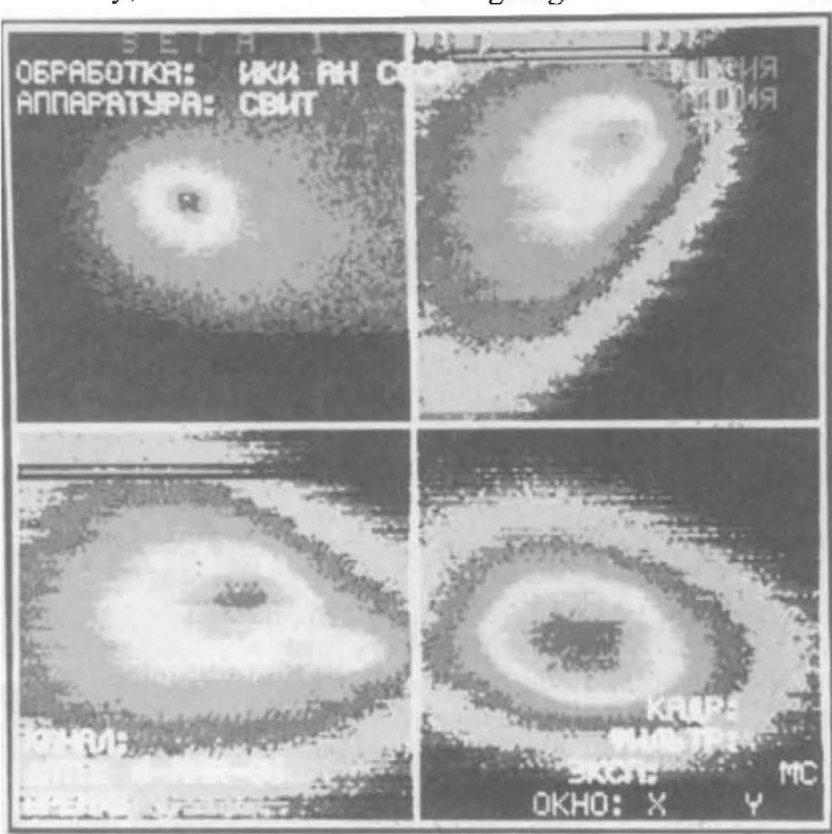

symposia. The experimentalists have been crammed with their microprocessors into a ground-floor room about $30 \mathrm{~m}^{2}$ dominated by a full-scale model of Vega. Each experiment has been able to monitor its own data stream in real time; most, almost as a matter of pride, have thought it seemly to produce an instant analysis and interpretation as well. The Hungarian image processers were back-to-back with the Austrian recorders of the magnetic field. The Czechoslovak and US dust particle experiments were within whispering distance of each other (with the West Germans across the corridor). The French team taking ultraviolet spectra have been almost inaccessible in their corner.

The other symposium, on the floor above, has been for dignitaries, and has been recognizable by the bottles of mineral water on the tables. (Local opinion holds that "something is going to happen" when the bottles are changed; "they know we'd drink them if they left them there too long".) The dignitaries have included Dr Fred Whipple, lately retired from the Harvard-Smithsonian Astrophysical Observatory, the originator of the dirty snowball picture, Dr Thomas Donahue, chairman of the US National Academy's Space Sciences Board, delegations from the European Space Agency (ESA), the US National Aeronautics and Space Administration (NASA) and from the Space Sciences Institute at the University of Tokyo and vice-presidents of the Soviet Academy, who dropped in from time to time.

During both encounters with Halley's comet, this symposium has been kept thoroughly up to date by a display on two substantial video screens of data as re-
False-colour images of Halley's comet (shown here in black and white) from Vega 1 at around the time of closest approach at 07.20 GMT on 6 March, with four different filters. Although the top left-hand image shows a small peak of intensity (which appears white) near the centre, this cannot be identified with the nucleus of the comet, but is rather an artefact of the colour-coding system. The double structure of the image immediately below, in the near infrared, may signify the jet of dust encountered by Vega 1 . The distance across each frame is about $1,500 \mathrm{~km}$. 